\title{
Phantom Field and the Fate of Universe
}

\author{
M. Sam* \\ IUCAA, Post Bag 4, Ganeshkhind, \\ Pune $41100 \%$, India. \\ Alexey Toporensky \\ Sternberg Astronomical Institute, Universitetski Prospect, 13, Moscow 119899, Russid
}

\begin{abstract}
In this paper we analyze the cosmological dynamics of phantom field in a variety of potentials unbounded from above. We demonstrate that the nature of future evolution generically depends upon the steepness of the phantom potential and discuss the fate of Universe accordingly.

PACS numbers: $98.80 . \mathrm{Cq}, 98.80 . \mathrm{Hw}, 04.50 .+\mathrm{h}$
\end{abstract}

\section{INTRODUCTION}

There is a growing evidence that Universe is undergoing accelerated expansion at present. The observations related to supernova, $\mathrm{CMB}$ and galaxy clustering all together strongly point towards the compelling possibility of late time accelerated expansion of Universe. The acceleration of Universe can be accounted for either by modifying the left hand side of Einstein equations or by supplementing the energy momentum tensor by an exotic matter with negative pressure, popularly known as dark energy. In past few years there have been tremendous efforts in modeling the dark energy. They include scalar field models, some models of brane worlds and specific compactification schemes in string theory which have been shown to mimic the dark energy like behaviour. A wide variety of scalar field models have been conjectured for this purpose including quintessence 1], K-essence 2], tachyonic scalar fields with the last one being originally motivated by string theoretical ideas. All these models of scalar field lead to the equation of state parameter $w$ greater than or equal to minus one. However, the recent observations do not seem to exclude values of this parameter less than minus one 57]. It is therefore important to look for theoretical possibilities to describe dark energy with $w<-1$ called phantom energy $3,4,5,6,7,8,9$, 10, 11, 12, 13, 14, 15, 16, 17, 18, 19, 20, 21, 22, 23, 24, $25,26,27,28,29,30,31,32,33,34,35,36,37,38,39]$. Specific models in brane world or non-minimally coupled scalar fields may lead to phantom energy 40, 41]. In our opinion, the simplest alternative is provided by a phantom field, scalar field with negative kinetic energy. Such a field can be motivated from S-brane constructs in string theory 42, 43, 44, 45, 46, 47, 48, 49]. Historically, phantom fields were first introduced in Hoyle's version of the Steady State Theory. In adherence to the Perfect Cosmological Principle, a creation field (C-field) was for the

* On leave from: Department of Physics, Jamia Millia, New Delhi110025; Electronic address: sami@iucaa.ernet.in

${ }^{\dagger}$ Electronic address: lesha@sai.msu.ru first time introduced [50] to reconcile with homogeneous density by creation of new matter in the voids caused by the expansion of Universe. It was further refined and reformulated in the Hoyle and Narlikar theory of gravitation 51] (see also Ref. 52] on the similar theme). Though the quantum theory of phantom fields is problematic [53], it is nevertheless interesting to examine the cosmological consequences of these fields at the classical level.

Models with constant $w<-1$ lead to unwanted future singularity called big rip [54]. This singularity is characterized by the divergence of scale factor after a finite interval of time. Infact there is no profound reason to assume that the equation of state parameter $w$ would remain constant in future if it has been changing all the way since the end of inflation to date. Keeping this in mind, specific scalar field models were proposed to avoid the cosmic doomsday 15,26 . It requires a special class of phantom field potentials with local maximum. In this paper we examine the nature of future evolution of Universe with a phantom field evolving in a more general class of potentials. The future course of evolution is shown to critically dependent on the steepness of the underlying potential.

\section{COSMOLOGICAL DYNAMICS IN THE PRESENCE OF PHANTOM FIELD}

The Lagrangian of the phantom field minimally coupled to gravity and matter sources is given by 20, 26.

$$
\mathcal{L}=(16 \pi G)^{-1} R+\frac{1}{2} g^{\mu \nu} \partial \phi_{\mu} \partial \phi_{\nu}-V(\phi)+\mathcal{L}_{\text {source }}
$$

where $\mathcal{L}_{\text {source }}$ is the remaining source term (matter, radiation) and $V(\phi)$ is the phantom potential. The kinetic energy term of the phantom field in (1) enters with the opposite sign in contrast to the ordinary scalar field (we employ the metric signature,,,,-+++$)$. In a spatially flat FRW cosmology, the stress tensor that follows from (11) acquires the diagonal form $T_{\beta}^{\alpha}=\operatorname{diag}(-\rho, p, p, p)$ where the pressure and energy density of field $\phi$ are given by

$$
\rho_{\phi}=-\frac{\dot{\phi}^{2}}{2}+V(\phi), \quad p_{\phi}=-\frac{\dot{\phi}^{2}}{2}-V(\phi) .
$$


The corresponding equation of state parameter is now given by

$$
w \equiv \frac{p_{\phi}}{\rho_{\phi}}=\frac{\frac{\dot{\phi}^{2}}{2}+V(\phi)}{\frac{\dot{\phi}^{2}}{2}-V(\phi)} .
$$

For $\rho_{\phi}>0, w<-1$.

The equations of motion which follow from (1) are

$$
\begin{gathered}
\dot{H}=-\frac{1}{2 M_{p}^{2}}\left(\rho_{b}+p_{b}-\dot{\phi}^{2}\right) \\
H^{2}=\frac{1}{3 M_{p}^{2}}\left(\rho_{b}+\rho_{\phi}\right) \\
\ddot{\phi}+3 H \dot{\phi}=V^{\prime}(\phi)
\end{gathered}
$$

where the background energy density due to matter and radiation is given by

$$
\rho_{b}=\frac{\rho_{R}^{i}}{a^{4}}+\frac{\rho_{m}^{i}}{a^{3}}
$$

As in the previous work 15, 26 we start with the phantom field energy density being dominated by the density of the ordinary matter. In this situation, an initial kinetic term of the field rapidly decreases due to the friction term $3 H \dot{\phi}$ in (6) and as a result the phantom field "freezes" at some point waiting for the moment its energy density becomes comparable with the matter (see figure 1). Subsequently, the field dynamics "switches on" and the nature of future evolution then depends on the shape of the phantom field potential $V(\phi)$.

The case of potentials with a local maximum was already described in [15, 26]. The phantom field asymptotically reaches the maximum with a possible oscillating transient stage. The future asymptotic regime is the DeSitter one with $\omega \rightarrow-1$ and $\Lambda=V_{\max } / M_{p}^{2}$.

Potentials other than these can lead to a variety of future evolutionary regimes. We begin with a simplest potential of a massive phantom field

$$
V(\phi)=m^{2} \phi^{2}
$$

When the phantom energy begins to dominate and $\dot{\phi}$ is small, we have, see equation (5) and (6)

$$
H \simeq \frac{m \phi}{\sqrt{3 M_{p}^{2}}}, \quad \dot{\phi} \simeq \frac{2 m M_{p}}{\sqrt{3}}
$$

Once such a regime is established its stability is guaranteed (see figure 2). Indeed, the ratio $\dot{\phi}^{2} / 2 V$ of the phantom field is inversely proportional to $\phi^{2}$ and tends to zero making the kinetic term subdominant forever. This regime is analogous to the slow-roll regime for a normal scalar field and can be named as "slow-climb". The effective equation of state tends to the De-Sitter one (see figure 3) with a slow growing energy density(figure $1)$. As a result, the final state of such Universe is formally different from both the De-Sitter and the "big rip" outcome - it would take infinite time to reach an infinite energy density in this case. However, such Universe will steadily reach the Planck density for the finite time and the classical physics breaks down here.

It can easily be checked that the condition $\dot{\phi}^{2} / 2 V \rightarrow 0$ is satisfied for any potential, which asymptotically reduces to power-law. Indeed, if the kinetic term is subdominant, equation (6) gives

$$
\dot{\phi}=\frac{V^{\prime}(\phi)}{3 H}
$$

In the regime of phantom field dominance we have

$$
\begin{gathered}
H^{2}=\frac{V(\phi)}{3 M_{p}^{2}} \\
\left(\frac{\dot{\phi}^{2}}{2}\right) \frac{1}{V(\phi)}=\frac{M_{p}^{2}}{6}\left(\frac{V^{\prime 2}}{V^{2}}\right)
\end{gathered}
$$

It is clear from (12) that $\dot{\phi}^{2} / 2 V$ is proportional to $\phi^{-2}$ for any power-law potential $V(\phi) \sim \phi^{\alpha}$.

However, the formally "slow climb" regime for steeper power-law potentials becomes the regime of a fast growth of the phantom field. The potential $V=\lambda \phi^{4}$ leads to

$$
H \simeq \frac{\sqrt{\lambda} \phi^{2}}{\sqrt{3 M_{p}^{2}}}, \quad V^{\prime}=4 \lambda \phi^{3}
$$

corresponding to

$$
\dot{\phi} \simeq 4 M_{p} \sqrt{\frac{\lambda}{3}} \phi
$$

which results in exponential growth of $\phi$. A bigger power index $\alpha$ leads to a "classical big rip" when a formally infinite energy density is reached during a finite time. Indeed, the general "slow-climb" equation for the phantom field with a power-law potential

$$
\dot{\phi} \simeq \phi^{\alpha / 2-1}
$$

in case of $\alpha>4$ leads to

$$
\phi \simeq\left(t_{0}-t\right)^{2 /(4-\alpha)}
$$

For completeness we note that asymptotically flat potentials, like $V(\phi)=A\left(1-\exp \left(-c \phi^{2}\right)\right)$ also lead to a "slow climb" but with asymptotically De-Sitter like behavior $\left(\Lambda=V(\infty) / M_{p}^{2}\right)$.

The approximation $\dot{\phi}^{2} / 2 V \rightarrow 0$ breaks down for exponential and steeper potentials. In case of exponential potentials 21], Hao and $\mathrm{Li}$ found an attractor solution with $\omega$ tending to a constant value less then -1 making the "big rip" inevitable. 


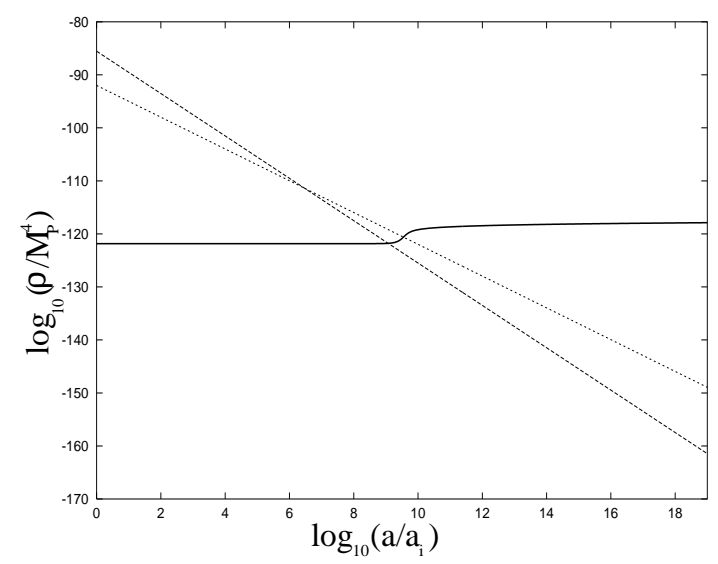

FIG. 1: Evolution of energy density is plotted against the scale factor. Solid line corresponds to the phantom field energy density in case of the model described by the potential: $V(\phi)=m^{2} \phi^{2}$ with $m \simeq 10^{-60} M_{p}$. The dashed and dotted lines correspond to energy density of radiation and matter. Initially, the phantom field mimics the cosmological constant like behaviour and its energy density is extremely subdominant to the background (the system is numerically evolved starting from the radiation domination era with $\rho_{r}=1 \mathrm{MeV}^{4}$ ) and remains to be so for most of the period of evolution. The phantom field $\phi$ continues in the state with $w=-1$ till the moment $\rho_{\phi}$ approaches $\rho_{b}$. The background ceases now to play the leading role (becomes subdominant) and the phantom field takes over and starts climbing up the potential fast. At late times, the field energy density catches up with the background, overtakes it and starts growing $(w<-1)$ and drives the current (the value of the scale factor $a \simeq 4 \times 10^{9}$ corresponds to the present epoch) accelerated expansion of the Universe with $\Omega_{\phi} \simeq 0.7$ and $\Omega_{m} \simeq 0.3$. Initial value of the field was tuned to get the present values of $\Omega$. The field then enters into the slow climbing regime allowing the slowing down of growth of $\rho_{\phi}$ and making $w \rightarrow-1$ asymptotically which is an attractor in this case.

When we turn to potentials steeper than an exponential, like $V(\phi)=\exp \left(c \phi^{2}\right)$ or potentials with an infinite potential wall, we get another type of a future singularity. It is evident from equation (12) that the kinetic term grows faster than the potential one, and at a particular epoch becomes important. However, $\rho_{\phi}=V(\phi)-\dot{\phi}^{2} / 2$ always grows as the equation (44) in the epoch of phantom dominance prevents $H$ and correspondingly $\rho_{\phi}$ (see equation (5) from decreasing. As a result, both potential and kinetic terms increase rapidly with less rapid increase of $\rho_{\phi}$. Our numerical studies indicate that the ratio of kinetic and potential terms tends to -1 and the state equation parameter $\omega$ blows up to $-\infty$ at some finite value of the scale factor $a$ (figure 3). Potential and kinetic terms of the phantom field also become infinite at this moment. This type of cosmological singularity, when equations of motion become singular at a finite value of scale factor are already known in some models of brane worlds [55], tachyonic field 36] and Gauss-Bonnet cosmol-

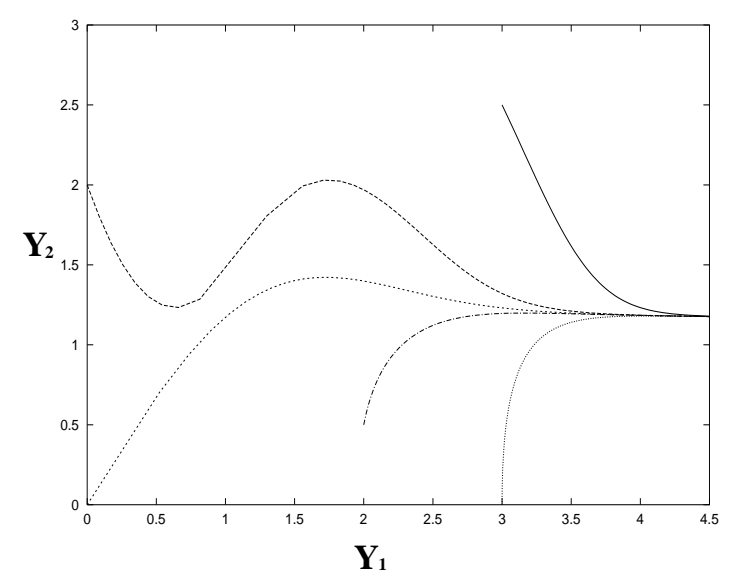

FIG. 2: Display of the phase portrait (plot of $Y_{2} \equiv \dot{\phi} / M_{p}^{2}$ versus $Y_{1} \equiv \phi / M_{p}$ ) for the phantom field in case of the quadratic potential $V(\phi)=m^{2} \phi^{2}$. The figure shows that the trajectories starting anywhere in the phase space move towards a configuration(slow climb regime) with $\dot{\phi} \rightarrow$ const asymptotically(the convenient choice of mass parameter in the potential used here corresponds to the value of the const equal to $2 / \sqrt{3}$ ). This picture is drawn in absence of the background energy density and allows to probe a wider class of initial conditions; the asymptotic regime is independent of the background.

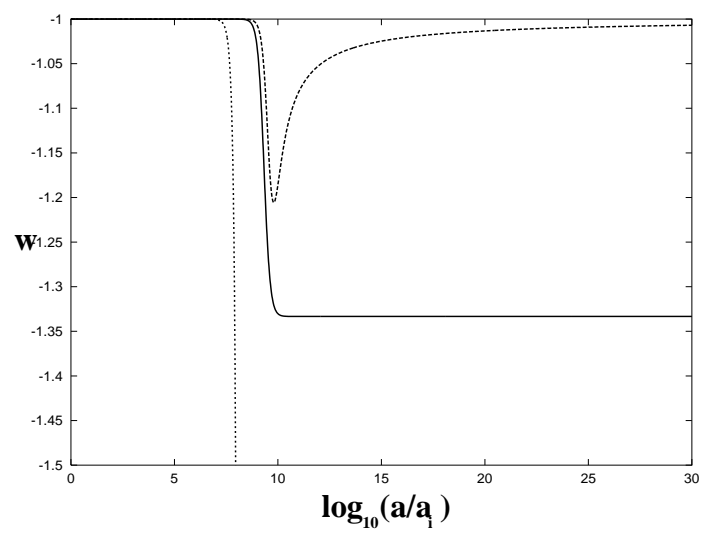

FIG. 3: Equation of state parameter $w$ for the phantom field is plotted against the scale factor. The solid line corresponds to the exponential potential $V(\phi) \sim e^{\lambda \phi / M_{p}}$, the dotted line to $V(\phi) \sim e^{\lambda \phi^{2} / M_{p}^{2}}$ and the dashed line to $V(\phi) \sim \phi^{2}$. The exponential potential exhibits a critical behaviour in which case $w$ fast evolves to a constant value less than minus one (numerical value depends upon $\lambda$ which was taken to be equal to one for convenience). For potential steeper than the exponential $w$ fast evolves towards larger and larger negative values (dotted line) leading to a singularity at some finite value of the scale factor. In case of a less steeper potential than the exponential, the equation of state parameter $w$ first decreases fast and then subsequently turns back towards minus one and approaches it asymptotically (dashed line). 
ogy $[56]$. However, we should note that all these scenarios lead to divergence in the second derivatives of metrics. The matter content of the Universe and Hubble parameter remain finite in these models. In case of the phantom field, the energy density itself blows up at some finite value of $a$.

\section{CONCLUSIONS}

We have studied the possible future regimes of Universe with a phantom field. The opposite sign in the kinetic term pushes the field towards maximum of its potential. This feature leads to a cosmological singularity in the future for phantom field potentials unbounded from above. The nature of this singularity crucially depends upon steepness of the potential. The analysis presented above allows us to classify the future cosmological behavior in the following types:

- For asymptotically power-law potentials $V(\phi) \sim \phi^{\alpha}$ with $\alpha \leq 4$ we obtain $\omega \rightarrow-1$ and $\rho_{\phi} \rightarrow \infty$ for $t \rightarrow \infty$.
- For $\alpha>4$ though $\omega \rightarrow-1$, but the infinite energy density is reached during a finite time, referred to as big rip.

- For exponential potentials, $\omega$ tends to a constant value less than -1 again leading to a big rip singularity.

- Potentials steeper than exponential lead to a singularity with $w \rightarrow-\infty$ for a finite value of scale factor.

\section{Acknowledgments}

We thank N. Dadhich, Parampreet Singh and V. Sahni for useful discussions. We also thank A. Starobinsky, Yun-Song Piao and Ishwaree P. Neupane for comments. MS is thankful to T. Padmanabhan for helpful discussions. AT acknowledges support from IUCAA's 'Program for enhanced interaction with the Africa-Asia-Pacific Region'. AT is grateful to IUCAA for hospitality where this work was done.
[1] B. Ratra, and P.J.E. Peebles, Phys. Rev. D 37, 3406 (1988); C. Wetterich, Nucl. Phys. B 302, 668 (1988); J. Frieman, C.T. Hill, A. Stebbins, and I. Waga, (1995) Phys. Rev. Lett. 75, 2077; P.G. Ferreira and M. Joyce, Phys. Rev. D 58, 023503 (1998); I. Zlatev, L, Wang and P.J. Steinhardt Phys. Rev. Lett. 82, 896 (1999); T. D. Saini, S. Raychaudhury, Varun Sahni and A. A. Starobinsky, Phys.Rev.Lett. 85 (2000) 1162-1165.

P. Brax and J. Martin Phys. Rev. D 61, 103502 (2000); L.A. Ureña-López and T. Matos, Phys. Rev. D 62, 081302 (2000); T. Barreiro, E.J. Copeland and N.J. Nunes Phys. Rev. D 61, 127301 (2000); A. Albrecht and C. Skordis Phys. Rev. Lett. 84, 2076 (2000); V. Johri, astro-ph/0005608 V. Johri, astro-ph/0108247 V. JohJ. P. Kneller and L. E. Strigari, asto-ph/0302167 F. Rossati, hep-ph/0302159 V. Sahni, M. Sami and T. Souradeep, Phys.Rev. D65, 023518 (2002); M. Sami, N. Dadhich and Tetsuya Shiromizu, Phys.Lett. B568 (2003) 118-126 hep-th/0304187; M. Sami and T. Padmanabhan, Phys.Rev. D 67 (2003) 083509 hep-th/0212317.

[2] Armendariz-Picon, T. Damour, V. Mukhanov, Phys.Lett. B458 (1999) 209 hep-th/9904075; T. Chiba, T. Okabe and M. Yamaguchi, Physical Review D62, 023511 (2000).

[3] V. Sahni and A. Starobinsky, IJMPD 9, 373(2000) astro-ph/9904398.

[4] R.R. Caldwell, Phys.Lett. B54523-29(2002).

[5] L. Parker and A. Raval, Phys. Rev. D60, 063512(1999).

[6] T. Chiba, T. Okabe and M. Yamaguchi, Phys. Rev. D62, 023511(2000).

[7] B. Boisseau, G. Esposito-Farese, D. Polarski and A. A. Starobinsky, Phys. Rev. Lett.85, 2236, (2000).

[8] A. E. Schulz, Martin White, Phys.Rev. D64 (2001) 043514.
[9] V. Faraoni, Int. J. Mod. Phys. D64, 043514 (2002).

[10] I. Maor, R. Brustein, J. Mcmahon and P. J. Steinhardt, Phys. Rev. D65 123003(2002).

[11] V. K. Onemli and R. P. Woodard, Class. Quant. Grav. 19, 4607(2002).

[12] D. F. Torres, Phys. Rev. D66, 043522 (2002).

[13] S. Hannestad, E. Mortsell, Phys. Rev. D66, 063508 (2002).

[14] A. Melchiorri, L. Mersini, C. J. Odman, M. Trodden, astro-ph/0211522

[15] S. M. Carroll, M. Hoffman and M. Trodden, astro-ph/0301273

[16] R. Mainini, A.V. Maccio', S.A. Bonometto, A.Klypin, astro-ph/0303303

[17] P. H. Frampton, hep-th/0302007

[18] Jian-gang Hao and Xin-zhou Li, hep-th/0302100

[19] R. R Caldwell, M. Kamionkowski and N. N. Weinberg, astro-ph/0302506

[20] G. W. Gibbons, Phantom matter and the cosmological constant, hep-th/0302199

[21] Jian-gang Hao, Xin-zhou Li, hep-th/0303093

[22] Shin'ichi Nojiri and Sergei D. Odintsov, hep-th/0303117 Shin'ichi Nojiri and Sergei D. Odintsov hep-th/0304131

[23] Alexander Feinstein and Sanjay Jhingan, hep-th/0304069

[24] J. A. S. Lima, J. V. Cunha and S. Alcaniz, astro-ph/0303388

[25] A. Yurov, astro-ph/0305019

[26] Parampreet Singh, M.Sami, Naresh Dadhich,Phys.Rev. D 68 (2003) 023522 hep-th/0305110

[27] Jian-gang Hao, Xin-zhou Li, hep-th/0305207

[28] Shin'ichi Nojiri, Sergei D. Odintsov hep-th/0306212

[29] Mariusz P. Dabrowski, Tomasz Stachowiak, Marek Szydlowski hep-th/0307128 
[30] Dao-jun Liu, Xin-zhou Li, . hep-th/0307239

[31] L.P. Chimento, Ruth Lazkoz, gr-qc/0307111

[32] Jian-gang Hao, Xin-zhou Li astro-ph/0309746

[33] Xin-He Meng, Peng Wang, hep-ph/0311070 .

[34] Hrvoje Stefancic astro-ph/0310904 Hrvoje Stefancic, astro-ph/0311247

[35] Vinod B. Johri, astro-ph/0311293

[36] V. Gorini, A. Yu. Kamenshchik and U. Moschella, V. Pasquier, hep-th/0311111

[37] Yun-Song Piao, E Zhou, hep-th/0308080

[38] Ujjaini Alam, Varun Sahni, Tarun Deep Saini and A. A. Starobinsky, astro-ph/0311364

[39] T. Roy Choudhury and T. Padmanabhan, astro-ph/0311622

[40] V. Sahni, Yu Shtanov, astro-ph/0202346

[41] Valerio Faraoni, gr-qc/0307086

[42] Brett McInnes, hep-th/0305107

[43] Mattias N.R. Wohlfarth, hep-th/0307179

[44] N. Ohta, hep-th/0303238 N. Ohta, hep-th/0304172

[45] S. Roy, hep-th/0304084

[46] M. Gutperle, R. Kallosh and A. Linde, hep-th/0304225

[47] Chiang-Mei Chen, Pei-Ming Ho, Ishwaree P. Neupane and John E. Wang, hep-th/0304177

[48] Chiang-Mei Chen, Pei-Ming Ho, Ishwaree P. Neupane, Nobuyoshi Ohta and John E. Wang, hep-th/0306291

[49] Ishwaree P. Neupane, hep-th/0311071

[50] F. Hoyle, Mon. Not. R. Astr. Soc. 108, 372 (1948); 109 , 365 (1949).

[51] F. Hoyle and J. V. Narlikar, Proc. Roy. Soc. A282, 191 (1964); Mon. Not. R. Astr. Soc. 155, 305 (1972); 155, 323 (1972).

[52] Narlikar, J. V. and Padmanabhan, T., Phys. Rev.D32, 1928(1985).

[53] James M. Cline, Sangyong Jeon, Guy D. Moore, hep-ph/0311312

[54] A. Starobinsky, Grav. and Cosmol. 6, 157(2000) astro-ph/9912054.

[55] Yu.Shtanov and V. Sahni, Class.Quant.Grav. 19 (2002) L101-L107 gr-qc/0204040.

[56] Alexey Toporensky and Shinji Tsujikawa, Phys.Rev.D65:123509(2002). gr-qc/0202067

[57] It is argued in Refs. 38, 39] that recent observations favor $w<-1$. 